\title{
Partial Metabolic Response
}

National Cancer Institute

\section{Source}

National Cancer Institute. Partial Metabolic Response. NCI Thesaurus. Code C123609.

A decrease of metabolic tumor activity in all relevant lesions, based on a pre-defined threshold. 